\title{
Reactive power sharing in microgrid using virtual voltage
}

\author{
Eder A. Molina-Viloria ${ }^{1}$, John E. Candelo-Becerra ${ }^{2}$, Fredy E. Hoyos Velasco ${ }^{3}$ \\ ${ }^{1}$ Tecnologico de Antioquia, Institución Universitaria, Facultad de Ingeniería, Medellín, Colombia \\ ${ }^{2}$ Universidad Nacional de Colombia-Sede Medellín-Facultad de Minas-Departamento de Energía Eléctrica y Automática, \\ Medellín, Colombia \\ ${ }^{3}$ Universidad Nacional de Colombia-Sede Medellín-Facultad de Ciencias-Escuela de Física, Medellín, Colombia
}

\begin{tabular}{l}
\hline Article Info \\
\hline Article history: \\
Received Feb 5, 2020 \\
Revised Nov 9, 2020 \\
Accepted Nov 17, 2020 \\
\hline
\end{tabular}

Keywords:

Microgrid

Power transfer

Reactive power sharing

Virtual voltage

Voltage control

\begin{abstract}
The traditional droop control strategy has been applied previously in microgrids (MGs) to share accurately the active power. However, in some cases the result obtained when sharing reactive power is not the best, because of the parameters related to the distances from distributed generators (DGs) to the loads and the power variations. Therefore, this paper proposes a reactive power control strategy for a low voltage $\mathrm{MG}$, where the unequal impedance related to the distances between generators and loads requires adjustments to work with the conventional frequency and voltage droop methods. Thus, an additional coefficient is calculated from parameters of the network that relate the location of elements. The test is perfomed by simulations in the MATLAB-Simulink software, considering a three-node MG with three DGs and a load that can change power at different periods of time. The results show that it is possible to improve reactive power sharing between the DGs located in the MG according to the load changes simulated and to improve voltages with this method.
\end{abstract}

This is an open access article under the CC BY-SA license.

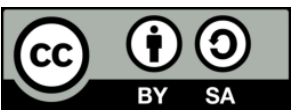

\section{Corresponding Author:}

Fredy E. Hoyos Velasco

Facultad de Ciencias-Escuela de Física

Universidad Nacional de Colombia, Sede Medellín

Carrera 65 Nro. 59A-110, Medellín, 050034, Colombia

Email: fehoyosve@unal.edu.co

\section{INTRODUCTION}

Microgrids (MGs) help relieve the stress of the main transmission systems, reduce electrical losses, and improve power quality of the systems as it is very effective to integrate renewable energy sources [1]. Some studies have been carried out in MGs considering different perspectives such as the need of compensation to improve power quality [2], power management and control [3, 4], protection [5], programming [6], and communication [7]. An MG can combine several renewable energy units, loads, and energy storage system [8]. Compared to the conventional distribution system, an MG can be operated connected to the power grid or island mode [9]. However, in low voltage MGs, the low capacity of reactive power supply from generation sources and compensators does not allow an exact distribution of reactive power to avoid overloads [10], because the impedance of the distribution feeder is mainly resistive [11]; in this case the droop control method is subject to poor transient stability, due to the poor coupling of reactive power between DG units when no additional inductance is present [11].

Line impedances and DG output impedances significantly affect the sharing of reactive power during the operating mode connected to the network and during the island mode, due to voltage drops [11]. Consequently, the concepts of droop control have been widely adopted to provide decentralized power, without relying on communications [12]. Although the frequency droop control technique can be used to 
achieve accurate real power distribution, voltage droop control commonly results in poor reactive power distribution [13]. This is due to the lack of coincidence in the voltage drops across the DG, which is induced by the mismatch in the impedance feeder and/or the differences in the powers of the units [14].

At present, the voltage controllers in the MGs are unable to share the reactive power demand among even identical inverters operating in parallel [15]. Some researchers have previously worked on this issue, as in [16], which propose an alternative controller for reactive power sharing between parallel inverters with nominal voltages. The method requires that each unit have a common load voltage measurement, which limits its applicability in more complex MG scenarios with multiple loads. In [17], the method improves the distribution of reactive power by changing the voltage bias based on conventional slope control, which is activated by a sequence of synchronization events through the communication network. In [18], it is illustrated the control strategy to improve the distribution of reactive power and decrease in a single-phase MG on the island. In [19], a distributed secondary control strategy is proposed, in which each generator uses the measurements of other DGs at each sample time for the control of frequency, voltage and reactive power. In [20], the restoration of frequency and voltage is presented as the secondary control in a hierarchical control of the MG. In these methods the nominal value of the frequency and voltage deviation is determined in the central control and then transmitted to the other DGs of the MG to restore them. Therefore, MG control is assumed centralized in this method.

In [21], a resistive capacitive output impedance (RC-Inverter) is proposed. This equivalent output impedance of the $\mathrm{RC}$ inverter is designed by the introduction of capacitive-resistive virtual impedances. This usually provides rapid reactive power for low MGs voltages and maintains system voltage stability. However, the method works by estimating the voltage drops with the virtual impedance, so the error in the estimate makes the method not work well and accurately. In [22] a voltage source inverter based on servo system is proposed, which is designed as a reference tracker. A new control is proposed to determine the set point of reactive power in each inverter to make its contribution in the distribution of reactive power; but it presents the problem of communication, which makes it very impractical when implemented in industrial cases.

In [23], a new decentralized model is proposed for distribution of reactive power and frequency restoration in an island system. The proposed method does not need communication link between the DG, synchronization between each DG is achieved by detecting load change with the Wavelet transform; but this model is very flawed, since it uses two compensators which act one after the other, after a certain time when a load change occurs a condition is necessary for the second compensator to act and it is necessary that at that time there is no another load change, which would be obsolete in the face of major changes as in real systems. In [24], a novel droop control method for voltage and frequency in autonomous MGs is proposed, by incorporating predictive mechanisms in the DG. However, this method fails to share reactive power between the different DGs.

Therefore, the references consulted show that the previous work has focused on the control of active and reactive powers in MGs in island mode and how the virtual voltage has not been considered based on the variable load voltage that is connect to the MG as part of the solution. Therefore, the objective of this work is to share reactive power in an MG using the concept of virtual voltage and how it improves voltage regulation better than the droop control. Therefore, the contributions in this article are: the virtual voltage variables that are calculated for each inverter based on the RMS value of the node voltage where the loads are connected. The exchange of reactive power is achieved in several nodes of a network, once there are some variations in loads.

\section{MATERIAL AND METHOD}

The P- $\omega$ and Q-V droop controls have been used successfully in MGs [25]. This method is simple and can perform the 'plug-and-play' feature without communication. The P- $\omega$ droop control is used to control and regulate the frequency of the electrical network to share efficiently the active power. In order to control and track the reference voltage, the voltage control loop uses a PI controller, where the reference voltage is given by the virtual voltage controller, and the current controller loop uses a $\mathrm{P}$ controller to adjust the inductance low pass filter current, more details can be seen in [26].

Consider the diagram presented in Figure 1, where we can calculate active and reactive powers flowing to the load from DGs. The active power and the reactive powers transferred from the inverter to the load is given by (1) and (2). The term $\mathrm{P}$ is active power, $\mathrm{Q}$ is reactive power, $\mathrm{E}$ is the voltage at the terminals of the DG, $\mathrm{R}$ is the resistive part of the line impedance, $\mathrm{X}$ is the inductance of the line impedance, and $\mathrm{V}$ is the voltage in the load node. These two equations consider the active and reactive power losses due to the inductanace associated to the network.

$$
P=\frac{1}{R^{2}+X^{2}}\left(R E^{2}-R E V \cos \delta+X E V \sin \delta\right)
$$




$$
Q=\frac{1}{R^{2}+X^{2}}\left(X E^{2}-X E V \cos \delta-R E V \sin \delta\right)
$$

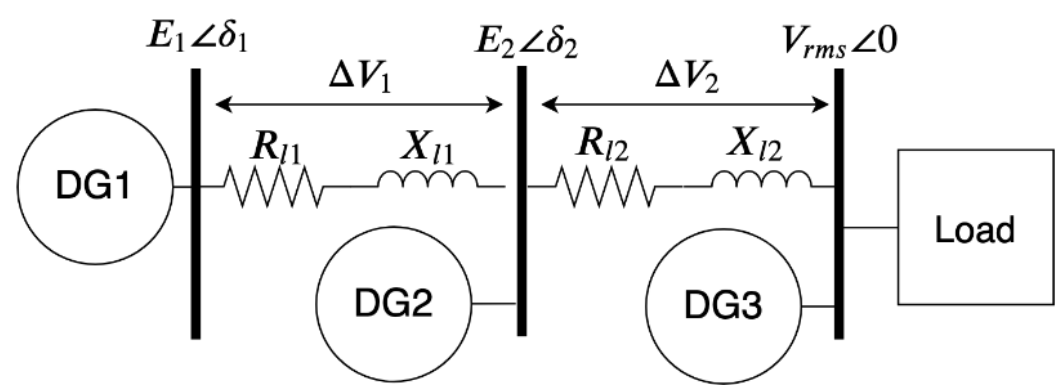

Figure 1. Simplified diagram of the MG

The controlled system has been presented in $[26,27]$. Therefore, a small signal model is derived for the proposed controller, in which the virtual voltage that is based on the RMS voltage of the load node V_rms, it is considered as the variable controller. By considering small disturbances around the equilibrium state defined by $(\Delta \delta \mathrm{e}, \mathrm{E}, \mathrm{V})$, equations (1)-(2) can be linearized around this point, then we have:

$$
\Delta P(s)=\left(\frac{\partial P}{\partial E_{V}}\right) \Delta E_{V}(s)+\left(\frac{\partial P}{\partial \delta}\right) \Delta \delta(s)
$$

We can express this last equation in terms of $k_{p e}$ and $k_{p s}$ as $\Delta P=k_{p e} \Delta E_{v}(s)+k_{p s} \Delta \delta(s)$. Similarly, the small disturbances around the equilibrium point are defined by $(\Delta \delta e, E, V)$ and the reactive power equation can be linearized around this point as expressed in (4).

$$
\Delta Q(s)=\left(\frac{\partial Q}{\partial E_{V}}\right) \Delta X_{V}(s)+\left(\frac{\partial Q}{\partial \delta}\right) \Delta \delta(s)
$$

In terms of $k_{q e}$ and $k_{q s}$, this last equation can be expressed as $\Delta Q(s)=K_{q e} \Delta E_{V}(s)+k_{q s} \Delta \delta(s)$; where the term $\Delta$ indicates the small-signal deviation of the variable around the equilibrium point and theses variations are expressed as $\Delta W=-k_{p} \Delta p$ and $\Delta E=K \Delta V_{r m s}$. The terms $k_{p e}, k_{p s}, K_{q e}$, and $k_{q s}$ are calculated around the equilibrium point, which is the sensitivity of the power flow to the virtual voltage and voltage angle regulation. Thus, the partial derivative of the active power based on the virtual is shown in the term as $k_{p e}$.

$$
k_{p e}=\left(\frac{\partial P}{\partial E_{V}}\right)=\frac{\left(2 R E_{V}-R V \cos \delta+X V \sin \delta\right)}{\left(R^{2}+X^{2}\right)}
$$

Similarly, we can obtain the partial derivative of the active power with respect to the angle and it is expressed as $k_{p s}$.

$$
k_{p s}=\left(\frac{\partial P}{\partial \delta}\right)=\frac{1}{R^{2}+X^{2}}\left(R E_{V} V \sin \delta+X E_{V} V \cos \delta\right)
$$
in (7) as $k_{q e}$.

Now, the partial derivative of the reactive power with respect to the virtual impedance is presented

$$
k_{q e}=\left(\frac{\partial Q}{\partial E_{V}}\right)=\frac{\left(2 X E_{V}-R V \sin \delta-X V \cos \delta\right)}{\left(R^{2}+X^{2}\right)}
$$

From the partial derivative of the reactive power with respect to the virtual impedance, (8) is obtained:

$$
k_{q s}=\left(\frac{\partial Q}{\partial \delta}\right)=\frac{1}{R^{2}+X^{2}}(X E V \sin \delta-R E V \cos \delta)
$$


Now, supposs that the real power and the reactive power are measured using a first-order low-pass filter, the cut-off frequency $W_{f}$ of the active power droop controller and the reactive power controller can be linearized around the same point [26].

$$
\begin{aligned}
& \Delta P_{\text {med }}=\frac{W_{f}}{s+W_{f}} \Delta P(s) \\
& \Delta Q_{\text {med }}=\frac{W_{f}}{s+W_{f}} \Delta Q(s)
\end{aligned}
$$

Therefore, it follows from (9), (10) that:

$$
\begin{aligned}
& \Delta W(s)=\frac{-K_{m} W_{f}}{s+W_{f}}\left(k_{p e} \Delta E_{v}(s)+k_{p s} \Delta \delta(s)\right) \\
& \Delta E(s)=\frac{-K_{v} W_{f}}{s+W_{f}} \Delta V_{r m s}(s)
\end{aligned}
$$

We can use the last term in (12) to replace it in (11), and obtain:

$$
\Delta W(s)=\frac{-K_{p} W_{f}}{S+W_{f}}\left[k_{p e}\left(\frac{-K_{v} W_{f}}{S+W_{f}} \Delta V_{r m s}(s)\right)+k_{p s} \Delta \delta(s)\right]
$$

Using (3)-(6), equation (14) can be obtained:

$$
\Delta V_{r m s}(s)=\left[\frac{\Delta w(s)-k_{p} k_{p d} \Delta \delta(s)}{k_{p} k_{p e} k_{v}}\right]
$$

when we replace the term $\Delta V_{r m s}(s)$ in (13) and we reorganize again, we obtain the expression (15):

$$
\Delta W(s)=\frac{-K_{p} w_{f}}{S+w_{f}}\left[k_{p e}\left(\frac{-K_{v} W_{f}}{S+W_{f}}\left[\frac{\Delta w(s)-k_{p} k_{p d} \Delta \delta(s)}{k_{p} k_{p e} k_{v}}\right]\right)+k_{p s} \Delta \delta(s)\right]
$$

Finally, by considering that $\Delta W(S)=S \Delta \delta(S)$, we can obtain a homogeneous equation that describes the free movement of the system for small disturbances around the equilibrium point $(\delta, \mathrm{E}, \mathrm{V})$. Equation (16) considers that $a=k_{p} k_{p e} k_{v}, b=2 w_{f} k_{p} k_{p e} k_{v}, c=k_{p}{ }^{2} k_{p \delta} w_{f} k_{p e} k_{v}$, and $d=2 w_{f}{ }^{2} k_{p}{ }^{2} k_{p s} k_{p e} k_{v}$.

$$
a S^{3} \Delta \delta(s)+b S^{2} \Delta \delta(s)+c S \Delta \delta(s)+d \Delta \delta(s)=0
$$

\section{RESULTS AND DISCUSSION}

The DGs presented in the MG of Figure 1 deliver active power to the loads including the effects of line impedances. Figures 2(a), 2(b) and 2(c) show the active power delivered by each DG (DG1, DG2, DG3), based on the strategy used, which are the strategy of droop control vs the virtual voltage RMS. The graphs show the comparison of the two control strategies and their behavior in the case of a load change in the MG node.

The active powers increase depending on the amount of electric load that are connected to the node. We can observe how the new proposed controller responds faster and better than the droop controller. It can be seen that both controllers manage to share the active power precisely, according to the connection and disconnection of different loads. We can also detail that the proposed control strategy manages to stabilize the active power much faster than the fixed value strategy. Furthermore, we can see how generators DG1, DG2 and DG3 share the active power with two different controllers in their inverters.

Figure 3(a) shows the proposed control strategy of the RMS voltage and Figure 3(b) the droop control strategy. We see that these strategies manage to share the active power precisely in the face of different load changes. So during the first 12 seconds we observe the active power consumption of approximately $2100 \mathrm{~W}$, as a result of which a load of $(10+\mathrm{j} 0.05) \Omega$ is connected. In the 12 -second period a load of $(15+\mathrm{j} 0.05) \Omega$ is connected, in this period the active power consumption of the load in the node is increased based on the consumption of the previous period, and it is possible to see that both control strategies respond very well to the connection of the new load, only that the proposed strategy manages to stabilize at a fixed value the active power much faster than the droop. 


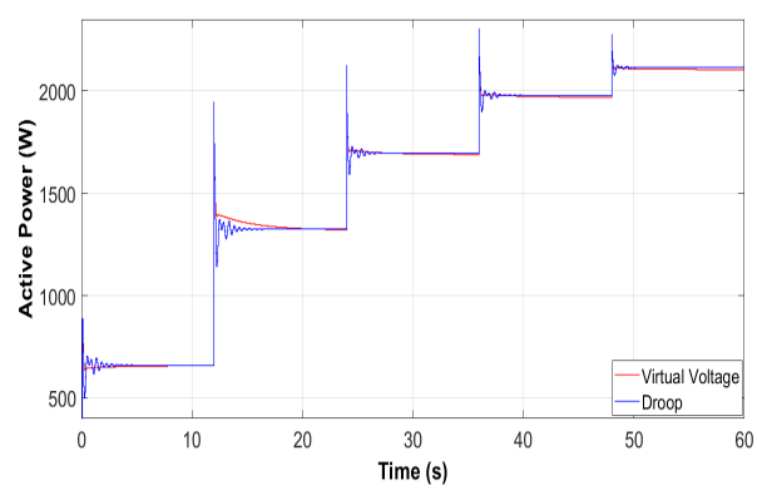

(a)

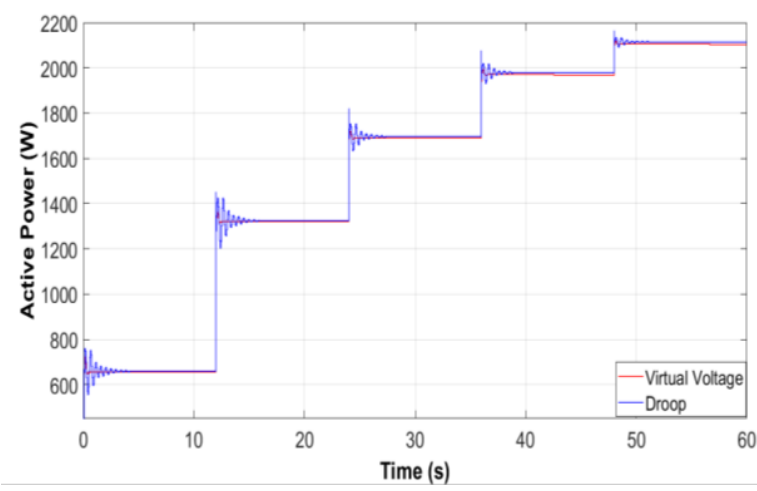

(b)

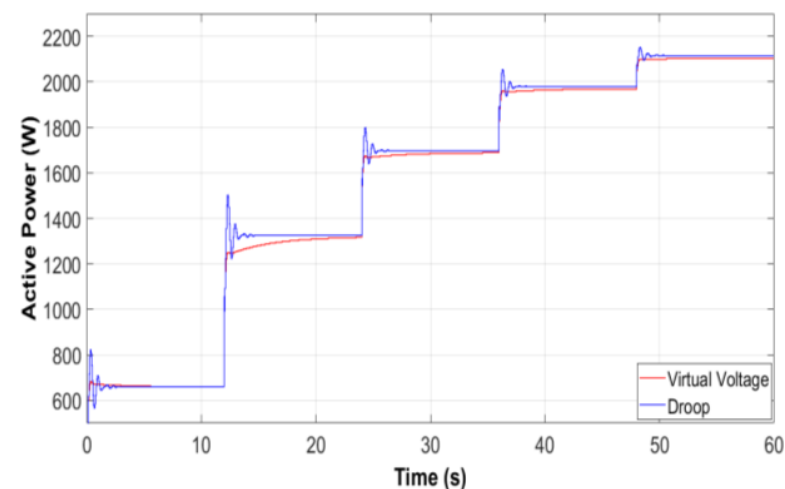

(c)

Figure 2. Active power obtained with the virtual voltage and droop control strategies; (a) DG1, (b) DG2, and (c) DG 3

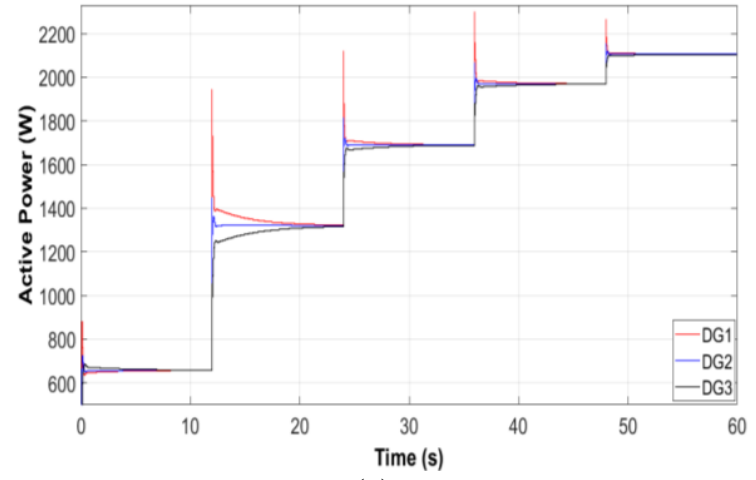

(a)

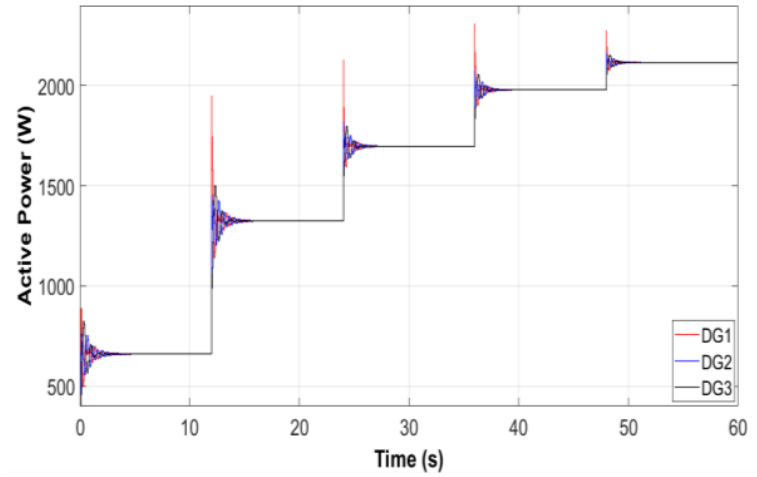

(b)

Figure 3. Active power of DGs obtained with the; (a) virtual voltage and (b) droop control strategies

In the 24 -second period a load of $(20+\mathrm{j} 0.08) \Omega$ is connected to the MG node, in this period an increase in power consumption is generated, but the control strategy acts immediately and recover the active power of the load at a stable value. In the course of 36 seconds a load $(25+\mathrm{j} 0.10) \Omega$ is connected, in this period a considerable increase in the active power consumption in the node is generated, but it is possible to see how the control strategy Proposal responds quickly and manages to stabilize the power value at a fixed value. In the course of 48 seconds, a load of $(50+\mathrm{j} 0.20) \Omega$ is connected, which causes the active power supplied by the three generators to increase based on the previous period. At this point we observe how both controllers respond exceptionally to the load change.

Moreover, two different control strategies in their respective inverters are presented in Figure 4(a) the proposed RMS voltage control strategy and Figure 4(b) the droop control strategy. We see that only the 
proposed control strategy manages to share the reactive power quickly and it is stable for different load changes and this is not achieved by the droop control. During the first 12 seconds we can see how a load of $(10+\mathrm{j} 0.05) \Omega$ is connected in this period of time and the proposed control strategy responds very well to the connection of the load, delivering a reactive power in proportion to the distance. In the period of 12 seconds to 24 seconds a load of $(15+\mathrm{j} 0.05) \Omega$ is connected, and the reactive power consumption of the load in the node is increased based on the consumption of the previous period. In this period of time DG1, DG2 and DG3 deliver different reactive powers that depend on the distance from the load to each generator.

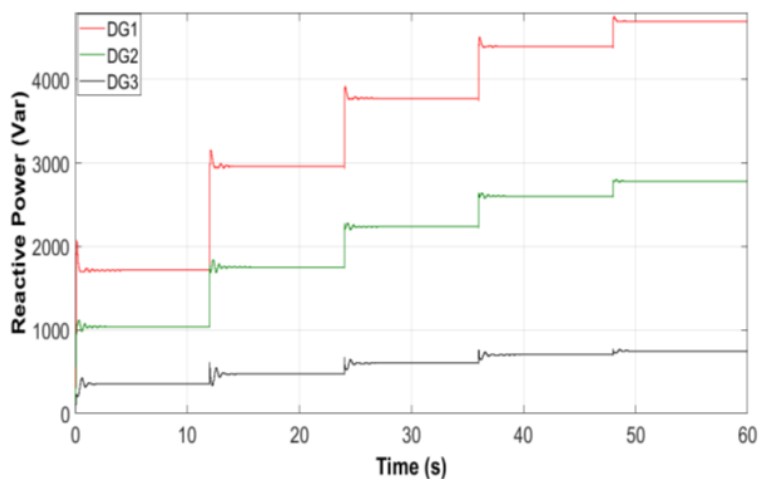

(a)

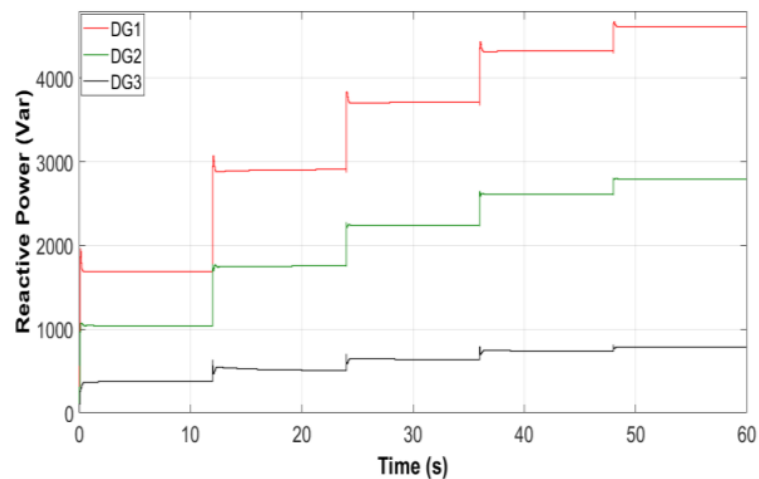

(b)

Figure 4. Reactive power of DGs obtained with the; (a) virtual voltage and (b) droop control strategies

A load of $(20+\mathrm{j} 0.08) \Omega$ is connected to the MG node in the period of 24 seconds to 36 seconds. In this period of time it is observed that DG1, DG2 and DG3 increase the reactive power delivered to the MG in different proportions, as a result of the new load on the MG. Over a period of 36 seconds to 48 seconds a load $(25+\mathrm{j} 0.10) \Omega$ is connected, which generates an increase in the consumption of reactive power in the MG, showing that the proposed control strategy responds quickly and manages to stabilize the power at a fixed value. In the course of 48 seconds the load $(50+j 0.20) \Omega$ is connected, which causes the reactive power supplied by the three generators to increase based on the previous period. In this period of 48 to 60 seconds, we can observe how the proposed control strategy responds much faster and manages to stabilize at a fixed value much faster than the droop control strategy.

In Figures 5(a), 5(b) and 5(c), we observe the reactive power of the three generators that conform the MG with the proposed controller and the droop controller, when different electrical loads are connected. Over the course of the simulation, we can see that the proposed control responds faster and stabilizes in less time and better than the droop control. Figure 6(a) shows the behavior of how the proposed and the droop controls response to the connection of different electrical loads over a period of time. Figure 6(b) shows the behavior of the RMS voltage at the node where the five electrical loads of the MG are connected.

The figure shows the RMS voltage of the proposed control strategy in blue and the droop control strategy in red. We can observe how the new proposed control responds faster and better than the droop control, to different load changes. The proposed control strategy allows the frequency of the three generators to stabilize at a single value, a few seconds after the load changes. Besides, the controller maintains the frequency close to a reference value and closer than the droop controller after load variations. These results show that when the connection of the second load is made, the node voltage drops sharply, but immediately it recovers the voltage to a close original value. Therefore, the proposed control maintains a stable value during all periods in which the electrical loads are connected and disconnected. However, when the droop control strategy is used, the voltage drops below the value obtained with the proposed control strategy, which is why we can conclude according to what has been observed as the proposed new control strategy responds faster and better than the droop controller.

Figures 7(a) and 7(b) show the active and reactive powers when multiple loads are connected and disconnected at different periods of time in a node of the MG. The active and reactive powers increase depending on the amount of loads that are connected to the node. We can observe that the active power and reactive power consumed by the loads is the same, independent of the control strategy used to regulate and keep the voltage stable in the node where the loads are connected and disconnected. 


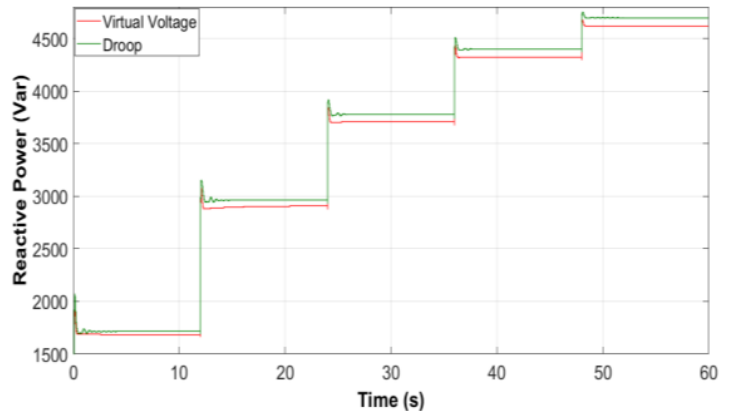

(a)

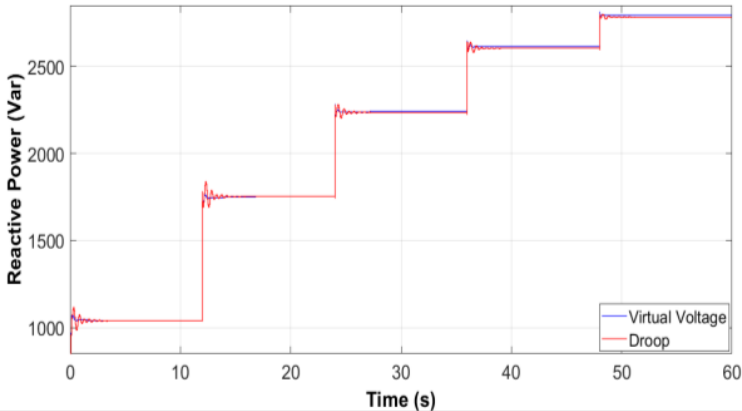

(b)

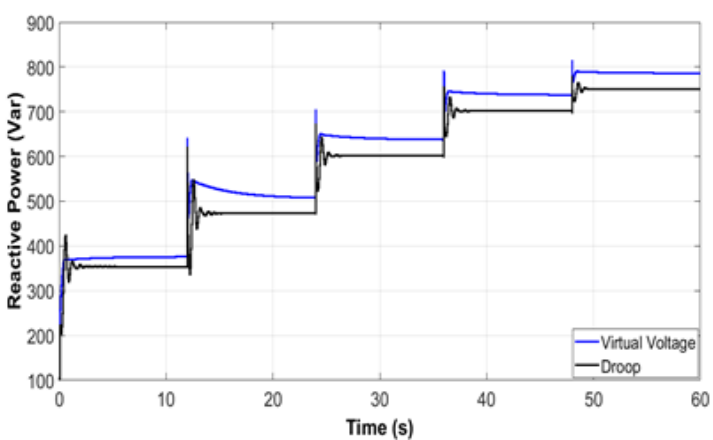

(c)

Figure 5. Reactive power of DGs obtained with the virtual voltage and droop control strategies; (a) DG1, (b) DG2, and (c) DG3

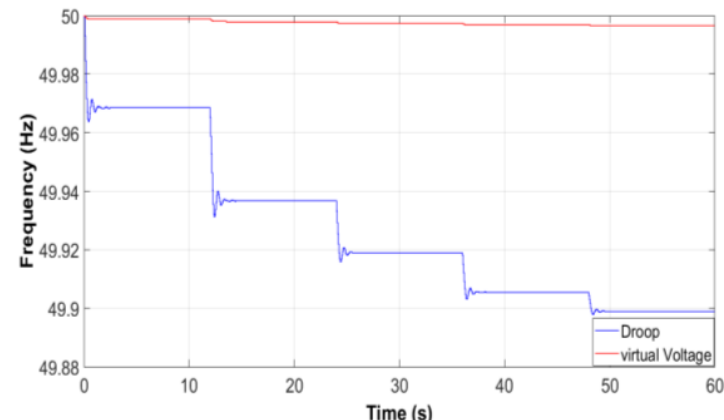

(a)

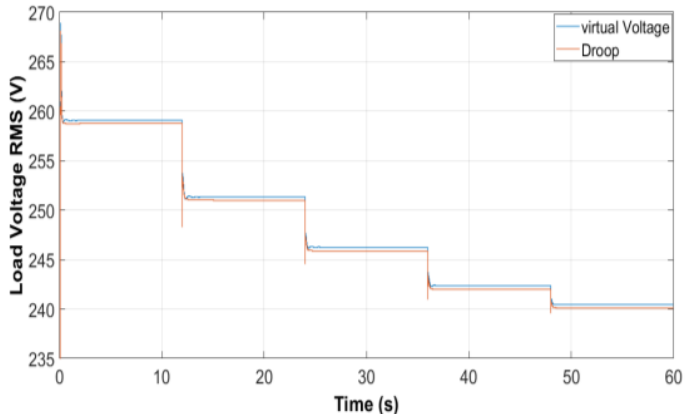

(b)

Figure 6. Results obtained with the virtual voltage and droop control strategies; (a) frequency and (b) load voltage

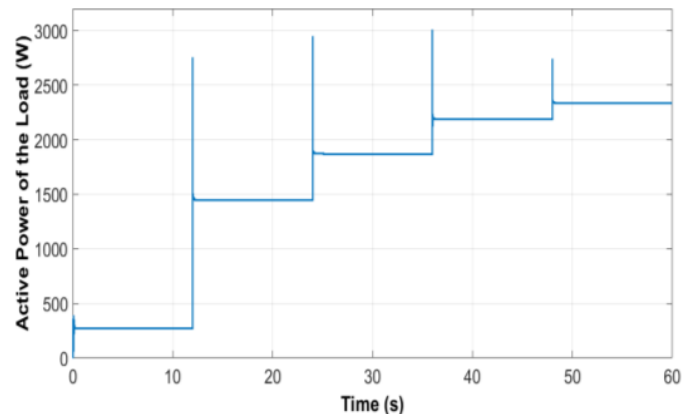

(a)

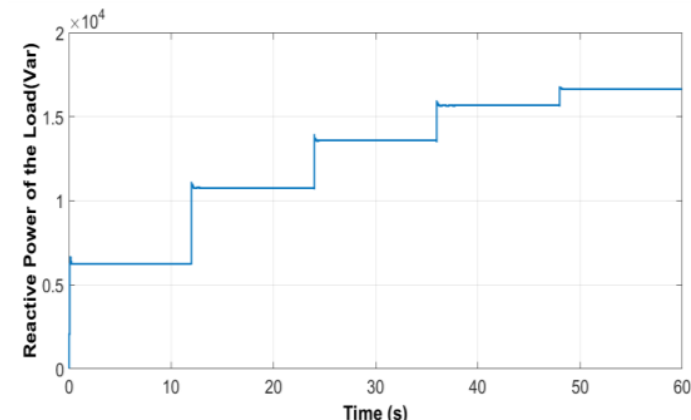

(b)

Figure 7. Response of the MG node for different load changes; (a) active power and (b) reactive power 


\section{CONCLUSION}

This paper presented a control strategy using a virtual RMS voltage to share the reactive power between different DGs in an MG, when electrical loads are connected at different periods of time. Results were obtained for a system with three DGs, with which it was possible to share reactive power between the DGs of the MG according to the different load changes. Therefore, at each moment the active power and the reactive power supplied by the DG increase, and this control strategy based on an adaptive virtual RMS voltage works successfully when it is related to the load node. The proposed control strategy effectively regulates the frequency close to the reference value and it also maintains the balances of the active and reactive powers.

\section{ACKNOWLEDGEMENTS}

The authors thank COLCIENCIAS, the Universidad Nacional de Colombia, and the Tecnologico de Antioquia, Institución Universitaria.

\section{REFERENCES}

[1] R. H. Lasseter, "MicroGrids," 2002 IEEE Power Engineering Society Winter Meeting, Conference Proceedings (Cat. No.02CH37309), New York, NY, USA, vol. 1, 2002, pp. 305-308.

[2] E. A. Molina, J. E. Candelo-Becerra, and F. E. Hoyos, "Control Strategy to Regulate Voltage and Share Reactive Power Using Variable Virtual Impedance for a Microgrid," Applied Sciences, vol. 9, no. 22, pp. 1-19, 2019.

[3] A. Bidram and A. Davoudi, "Hierarchical Structure of Microgrids Control System," IEEE Transactions on Smart Grid, vol. 3, no. 4, pp. 1963-1976, 2012.

[4] S. V. Iyer, M. N. Belur, and M. C. Chandorkar, "A Generalized Computational Method to Determine Stability of a Multi-inverter Microgrid," IEEE Transactions on Power Electronics, vol. 25, no. 9, pp. 2420-2432, 2010.

[5] T. S. Ustun, C. Ozansoy, and A. Ustun, "Fault current coefficient and time delay assignment for microgrid protection system with central protection unit," IEEE Transactions on Power Systems, vol. 28, no. 2, pp. 598-606, 2013.

[6] M. Tasdighi, H. Ghasemi, and A. Rahimi-Kian, "Residential Microgrid Scheduling Based on Smart Meters Data and Temperature Dependent Thermal Load Modeling," IEEE Transactions on Smart Grid, vol. 5, no. 1, pp. 349-357, 2014.

[7] T. Dragicevic, J. M. Guerrero, and J. C. Vasquez, "A Distributed Control Strategy for Coordination of an Autonomous LVDC Microgrid Based on Power-Line Signaling," IEEE Transactions on Industrial Electronics, vol. 61, no. 7, pp. 3313-3326, 2014.

[8] F. Katiraei and M. R. Iravani, "Power Management Strategies for a Microgrid with Multiple Distributed Generation Units," IEEE Transactions on Power Systems, vol. 21, no. 4, pp. 1821-1831, 2006.

[9] N. Pogaku, M. Prodanovic, and T. C. Green, "Modeling, Analysis and Testing of Autonomous Operation of an Inverter-Based Microgrid," IEEE Transactions on Power Electronics, vol. 22, no. 2, pp. 613-625, 2007.

[10] J. W. Simpson-Porco, Q. Shafiee, F. Dorfler, J. C. Vasquez, J. M. Guerrero, and F. Bullo, "Secondary Frequency and Voltage Control of Islanded Microgrids via Distributed Averaging," IEEE Transactions on Industrial Electronics, vol. 62, no. 11, pp. 7025-7038, 2015.

[11] Yun Wei Li and Ching-Nan Kao, "An Accurate Power Control Strategy for Power-Electronics-Interfaced Distributed Generation Units Operating in a Low-Voltage Multibus Microgrid," IEEE Transactions on Power Electronics, vol. 24, no. 12, pp. 2977-2988, 2009.

[12] J. Rocabert, A. Luna, F. Blaabjerg, and P. Rodríguez, "Control of Power Converters in AC Microgrids," IEEE Transactions on Power Electronics, vol. 27, no. 11, pp. 4734-4749, 2012.

[13] T. L. Vandoorn, J. C. Vasquez, J. De Kooning, J. M. Guerrero, and L. Vandevelde, "Microgrids: Hierarchical Control and an Overview of the Control and Reserve Management Strategies," IEEE Industrial Electronics Magazine, vol. 7, no. 4, pp. 42-55, 2013.

[14] J. Kim, J. M. Guerrero, P. Rodriguez, R. Teodorescu, and K. Nam, "Mode Adaptive Droop Control With Virtual Output Impedances for an Inverter-Based Flexible AC Microgrid," IEEE Transactions on Power Electronics, vol. 26, no. 3, pp. 689-701, 2011.

[15] J. A. P. Lopes, C. L. Moreira, and A. G. Madureira, "Defining Control Strategies for MicroGrids Islanded Operation," IEEE Transactions on Power Systems, vol. 21, no. 2, pp. 916-924, 2006.

[16] Q.-C. Zhong, "Robust Droop Controller for Accurate Proportional Load Sharing Among Inverters Operated in Parallel," IEEE Transactions on Industrial Electronics, vol. 60, no. 4, pp. 1281-1290, 2013.

[17] H. Han, Y. Liu, Y. Sun, M. Su, and J. M. Guerrero, "An Improved Droop Control Strategy for Reactive Power Sharing in Islanded Microgrid," IEEE Transactions on Power Electronics, vol. 30, no. 6, pp. 3133-3141, 2015.

[18] A. Micallef, M. Apap, C. Spiteri-Staines, J. M. Guerrero, and J. C. Vasquez, "Reactive Power Sharing and Voltage Harmonic Distortion Compensation of Droop Controlled Single Phase Islanded Microgrids," IEEE Transactions on Smart Grid, vol. 5, no. 3, pp. 1149-1158, 2014.

[19] Q. Shafiee, J. M. Guerrero, and J. C. Vasquez, "Distributed Secondary Control for Islanded Microgrids \&amp;\#x2014;A Novel Approach,” IEEE Transactions on Power Electronics, vol. 29, no. 2, pp. 1018-1031, 2014. 
[20] J. M. Guerrero, J. C. Vasquez, J. Matas, L. G. de Vicuna, and M. Castilla, "Hierarchical Control of DroopControlled AC and DC Microgrids-A General Approach Toward Standardization," IEEE Transactions on Industrial Electronics, vol. 58, no. 1, pp. 158-172, 2011.

[21] Y. Chen, A. Luo, J. Zhou, L. Bai, and C. Tu, "Rapid reactive power control method for parallel inverters using resistive-capacitive output impedance," 2013 1st International Future Energy Electronics Conference (IFEEC), Tainan, 2013, pp. 98-102.

[22] M. Eskandari, L. Li, and M. H. Moradi, "Decentralized Optimal Servo Control System for Implementing Instantaneous Reactive Power Sharing in Microgrids," IEEE Transactions on Sustainable Energy, vol. 9, no. 2, pp. 525-537, 2017.

[23] M. Kosari and S. H. Hosseinian, "Decentralized Reactive Power Sharing and Frequency Restoration in Islanded Microgrid," IEEE Transactions on Power Systems, vol. 32, no. 4, pp. 2901-2912, 2017.

[24] G. Lou, W. Gu, Y. Xu, M. Cheng, and W. Liu, "Distributed MPC-Based Secondary Voltage Control Scheme for Autonomous Droop-Controlled Microgrids," IEEE Transactions on Sustainable Energy, vol. 8, no. 2, pp. 792-804, 2017.

[25] K. De Brabandere, B. Bolsens, J. Van den Keybus, A. Woyte, J. Driesen, and R. Belmans, "A Voltage and Frequency Droop Control Method for Parallel Inverters," IEEE Transactions on Power Electronics, vol. 22, no. 4, pp. 1107-1115, 2007.

[26] Y. Hu, J. Xiang, Y. Peng, P. Yang, and W. Wei, "Decentralised control for reactive power sharing using adaptive virtual impedance," IET Generation Transmission and Distribution, vol. 12, no. 5, pp. 1198-1205, 2018.

[27] E. A. A. Coelho, P. C. Cortizo, and P. F. D. Garcia, "Small signal stability for single phase inverter connected to stiff AC system," Conference Record of the 1999 IEEE Industry Applications Conference. Thirty-Forth IAS Annual Meeting (Cat. No.99CH36370), Phoenix, AZ, USA, vol. 4, 1999, pp. 2180-2187. 